East Asian Mathematical Journal

Vol. 29 (2013), No. 1, pp. 13-22

http://dx.doi.org/10.7858/eamj.2013.002

\title{
MULTI-DIMENSIONAL LIU PROCESS, INTEGRAL AND DIFFERENTIAL
}

\author{
Cuilian You*, Huae Huo, and Weiging Wang
}

\begin{abstract}
As a fuzzy counterpart of stochastic calculus, fuzzy calculus including Liu integral and Liu formula were introduced. In order to deal with the problems with several fuzzy dynamic factors, Liu process, Liu integral and Liu formula are extended to the case of multi-dimensional in this paper.
\end{abstract}

\section{Introduction}

The concept of fuzzy set was introduced by Zadeh [22] via membership function in 1965. In order to measure a fuzzy event, a self-duality credibility measure was proposed by Liu and Liu [14] in 2002. Later, Li and Liu [10] provided a sufficient and necessary condition for credibility measure. As a branch of mathematics to study fuzzy phenomena, credibility theory was founded by Liu [11] and refined by Liu [12].

In the setting of credibility theory, a fuzzy variable was defined as a function from a credibility space to the set of real numbers.

As we know that there exist several dynamic fuzzy phenomena in real life, which are not enough to be described in one time, their evolutionary processes should be described repeatedly or continuously. Thus fuzzy process was investigated by many researches such as Kaleva [7, 8, 9], Pearson [16], Ding [3], Buckley [1], Lakshmikantham and Mohapatra [15]. Corresponding to Brownian motion, a fuzzy process was introduced by Liu in a seminar during summer holidays of 2007, then a fuzzy integral and a chain rule were given by Liu [13]. Later, the fuzzy process, fuzzy integral and chain rule were renamed as Liu process, Liu integral and Liu formula due to their importance and usefulness, just as Brownian motion, Ito integral and Ito formula. Up to now, many researches

Received March 30, 2012; Accepted December 17, 2012.

2000 Mathematics Subject Classification. 34A12.

Key words and phrases. fuzzy variable; fuzzy process; fuzzy integral.

This work was financially supported by Natural Science Foundation of China Grant No. 11201110 and 11101115, and Natural Science Foundation of Hebei Province No. A2011201007, and Outstanding Youth Science Fund of the Education Department of Hebei Province No. Y2012021.

*Corresponding author. 
have been done in this field. Qin [19] extended Liu process, Liu integral and Liu formula to the case of complex. Moreover, Dai [2] proved that Liu process is Lipschitz continuous and has finite variation. Based on these results, some applications of Liu process were made. Under the assumption that stock price is modeled by geometric Liu process, a basic stock model, which is called Liu's stock model later was proposed by Liu [13]. For different application background, two extended stock models were given by Gao [4] and Peng [17], respectively. By studying Liu stock model, Qin and Li [20] deduced the European option pricing formula, and it was applied to the problem of trading strategies by Qin and $\mathrm{Li}[21]$. Gao and Chen [6] extended Liu stock model to generalized case and obtained the corresponding European option pricing formula. By studying the stock model proposed by Gao [4], the European option pricing formula was derived by Gao [5]. Besides the application in mathematical finance, Liu process was also applied to fuzzy optimal control and an optimal equation was obtained by Zhu [23]. Qin [19] studied the production planning problem by a special fuzzy optimal control system.

In this paper, we will recall some basic concepts in fuzzy process. Then multi-dimensional fuzzy process and multi-dimensional Liu process will be introduced in Section 2. In Section 3, some properties of Liu integral will be discussed and then multi-dimensional Liu integral will be introduced. In Section 4, some differential theorems for multi-dimensional Liu process and high-order differential of function of Liu process are discussed. Finally, in Section 5, a brief summary is given.

\section{Multi-dimensional Liu Process}

Similar to stochastic process, a fuzzy process $X(t, \theta)$ is defined as a dual function of $t$ and $\theta$ such that $X\left(t^{*}, \theta\right)$ is a fuzzy variable for each $t^{*}$, where $t$ is time and $\theta$ is a point in credibility space $(\Theta, \mathcal{P}, \mathrm{Cr})$. For any fixed point $\theta \in \Theta, X\left(t, \theta^{*}\right)$ is a function of $t$, which is called a sample path of $X(t, \theta)$. For simplicity, we use the symbol $X_{t}$ to replace $X(t, \theta)$ in the following section.

A fuzzy process $X_{t}$ is called continuous if the sample paths of $X_{t}$ are all continuous functions of $t$ for almost all $\theta \in \Theta$.

Definition 1. (Liu [13]) A fuzzy process $X_{t}$ is said to have independent increments if $X_{t_{1}}-X_{t_{0}}, X_{t_{2}}-X_{t_{1}}, \cdots, X_{t_{k}}-X_{t_{k-1}}$ are independent fuzzy variables for any times $t_{0}<t_{1}<\cdots<t_{k}$. A fuzzy process $X_{t}$ is said to have stationary increments if, for any given $s>0, X_{t+s}-X_{t}$ are identically distributed fuzzy variables for all $t$.

As a special fuzzy process, Liu process was defined by Liu [13].

Definition 2. (Liu [13]) A fuzzy process $C_{t}$ is said to be a Liu process if

(i) $C_{0}=0$,

(ii) $C_{t}$ has stationary and independent increments,

(iii) every increment $C_{t+s}-C_{s}$ is a normally distributed fuzzy variable with 
expected value et and variance $\sigma^{2} t^{2}$ whose membership function is

$$
\mu(x)=2\left(1+\exp \left(\frac{\pi|x-e t|}{\sqrt{6} \sigma t}\right)\right)^{-1},-\infty<x<+\infty .
$$

The Liu process is said to be standard if $e=0$ and $\sigma=1$. Dai [2] stated Liu process is Lipschitz continuous and has finite variation.

An $m$-dimensional fuzzy process is defined as a function from $T \times(\Theta, \mathcal{P}, \mathrm{Cr})$ to the set of $m$-dimensional real vectors. Next, the definition of a multidimensional Liu process is given.

Definition 3. If $C_{i t}, i=1,2, \cdots, m$ are Liu processes, then $\boldsymbol{C}_{t}=\left(C_{1 t}, C_{2 t}\right.$, $\left.\cdots, C_{m t}\right)^{T}$ is called an $m$-dimensional Liu process.

Especially, an $m$-dimensional Liu process $\boldsymbol{C}_{t}=\left(C_{1 t}, C_{2 t}, \cdots, C_{m t}\right)^{T}$ is said to be standard if $C_{i t}$ are standard Liu processes for all $i$.

A fuzzy process $X_{t}=\exp \left(C_{t}\right)$ is called a geometric Liu process if $C_{t}$ is a Liu process. Geometric Liu process is expected to model stock prices. Multidimensional geometric Liu process is introduced to model stocks prices when there exist several stocks in the market at the same time.

Definition 4. Let $\left(C_{1 t}, C_{2 t}, \cdots, C_{m t}\right)^{T}$ be an $m$-dimensional Liu process, and $a_{i j}$ fuzzy processes for $i=1,2, \cdots, n$ and $j=1,2, \cdots, m$. Then the fuzzy process

$$
\boldsymbol{X}_{t}=\left(\exp \left(\sum_{j=1}^{m} a_{1 j} C_{j t}\right), \exp \left(\sum_{j=1}^{m} a_{2 j} C_{j t}\right), \cdots, \exp \left(\sum_{j=1}^{m} a_{n j} C_{j t}\right)\right)^{T}
$$

is called an $n$-dimensional geometric Liu process.

In the following two sections, we will discuss multi-dimensional Liu integral and multi-dimensional Liu differential, which are the extensions of Liu integral and Liu differential.

\section{Multi-dimensional Liu Integral}

Let $C_{t}$ be a standard Liu process, and let $\mathrm{d} t$ be an infinitesimal time interval. Then $\mathrm{d} C_{t}=C_{t+d t}-C_{t}$ is a fuzzy process, where $\mathrm{d} C_{t}$ is a normally distributed fuzzy variable with

$$
E\left[\mathrm{~d} C_{t}\right]=0, \quad V\left[\mathrm{~d} C_{t}\right]=\mathrm{d} t^{2}, E\left[\mathrm{~d} C_{t}^{2}\right]=\mathrm{d} t^{2}, \quad V\left[\mathrm{~d} C_{t}^{2}\right] \approx 7 \cdot \mathrm{d} t^{4} .
$$

Definition 5. (Liu Integral, Liu [13]) Let $X_{t}$ be a fuzzy process and let $C_{t}$ be a standard Liu process. For any partition of closed interval $[a, b]$ with $a=t_{1}<t_{2}<\cdots<t_{k+1}=b$, the mesh is written as $\triangle=\max _{1 \leq i \leq k}\left|t_{i+1}-t_{i}\right|$. Then the Liu integral of $X_{t}$ with respect to $C_{t}$ is defined as follows,

$$
\int_{a}^{b} X_{t} \mathrm{~d} C_{t}=\lim _{\triangle \rightarrow 0} \sum_{i=1}^{k} X_{t_{i}} \cdot\left(C_{t_{i+1}}-C_{t_{i}}\right)
$$


provided that the limitation exists almost surely and is a fuzzy variable. In this case, $X_{t}$ is called Liu integrable.

When there exists a multi-dimensional fuzzy process in a fuzzy dynamic system, it is natural to consider how to define the multi-dimensional integral?

Next, we will define a multi-dimensional Liu integral as follows:

Definition 6. (Multi-dimensional Liu Integral) Let $\boldsymbol{C}_{t}=\left(C_{1 t}, C_{2 t}, \cdots, C_{m t}\right)^{T}$ be an $m$-dimensional standard Liu process, and let $\mathcal{V}^{n \times m}$ denote the set of $n \times m$ matrices $v_{t}=\left[v_{i j t}\right]$, where each entry $v_{i j t}$ is a Liu integrable fuzzy process. Suppose $a<b$. If $v_{t} \in \mathcal{V}^{n \times m}$, the $m$-dimensional Liu integral is defined, using matrix notation

$$
\int_{a}^{b} v_{t} \mathrm{~d} \boldsymbol{C}_{t}=\int_{a}^{b}\left(\begin{array}{cccc}
v_{11 t} & v_{12 t} & \cdots & v_{1 m t} \\
v_{21 t} & v_{22 t} & \cdots & v_{2 m t} \\
\vdots & \vdots & & \vdots \\
v_{n 1 t} & v_{n 2 t} & \cdots & v_{n m t}
\end{array}\right)\left(\begin{array}{c}
\mathrm{d} C_{1 t} \\
\mathrm{~d} C_{2 t} \\
\vdots \\
\mathrm{d} C_{m t}
\end{array}\right)
$$

as the $n \times 1$ matrix whose $i$ th component is the following sum of Liu integrals:

$$
\sum_{j=1}^{m} \int_{a}^{b} v_{i j t} \mathrm{~d} C_{j t}
$$

Remark 3.1 Let $\boldsymbol{C}_{t}=\left(C_{1 t}, C_{2 t}, \cdots, C_{m t}\right)$ be an $m$-dimensional standard Liu process. Suppose $a<b$. If $v_{t}=\left(v_{1 t}, v_{2 t}, \cdots, v_{m t}\right)$, where $v_{i t}$ are Liu integrable fuzzy processes, the integral has the form

$$
\int_{a}^{b} v_{t} \mathrm{~d} \boldsymbol{C}_{t}=\sum_{i=1}^{m} \int_{a}^{b} v_{i t} \mathrm{~d} C_{i t}
$$

Remark 3.2 Let $C_{t}$ be a standard Liu process. Suppose $a<b$. If $v_{t}=$ $\left(v_{1 t}, v_{2 t}, \cdots, v_{n t}\right)^{T}$, where $v_{i t}$ are Liu integrable fuzzy processes, the integral has the form

$$
\int_{a}^{b} v_{t} \mathrm{~d} C_{t}=\left(\int_{a}^{b} v_{1 t} \mathrm{~d} C_{t}, \int_{a}^{b} v_{2 t} \mathrm{~d} C_{t}, \cdots \int_{a}^{b} v_{n t} \mathrm{~d} C_{t}\right)^{T} .
$$

\section{Multi-dimensional Fuzzy Differential}

Just like the role of Ito formula, Liu formula provides us with a tool to compute fuzzy calculus. In this section, some chain rules for fuzzy differential will be discussed.

Theorem 4.1. (Liu Formula, Liu [13]) Let $C_{t}$ be a standard Liu process, and let $h(t, x)$ be a continuously differentiable function. If fuzzy process $X_{t}$ is given 
by $\mathrm{d} X_{t}=u_{t} \mathrm{~d} t+v_{t} \mathrm{~d} C_{t}$, where $u_{t}, v_{t}$ are absolutely integrable fuzzy process and Liu integrable fuzzy process, respectively. Define $Y_{t}=h\left(t, X_{t}\right)$. Then

$$
\mathrm{d} Y_{t}=\frac{\partial h}{\partial t}\left(t, X_{t}\right) \mathrm{d} t+\frac{\partial h}{\partial x}\left(t, X_{t}\right) \mathrm{d} X_{t} .
$$

Example 4.1: The requirement that $h(t, x)$ is a continuously differentiable function in Theorem 4.1 is necessary, otherwise the result does not always hold. For example, let $C_{t}$ be a standard Liu process, and let

$$
h(t, x)=\left\{\begin{array}{cl}
x^{2} \sin \left(\frac{1}{x^{2}}\right), & x \neq 0 \\
0, & x=0 .
\end{array}\right.
$$

Then

$$
\frac{\partial h}{\partial x}=\left\{\begin{array}{cc}
2 x \sin \left(\frac{1}{x^{2}}\right)-\frac{2}{x} \cos \left(\frac{1}{x^{2}}\right), & x \neq 0 \\
0, & x=0 .
\end{array}\right.
$$

In this case,

$$
\begin{gathered}
X_{t}=h\left(t, C_{t}\right)=\left\{\begin{array}{cc}
C_{t}^{2} \sin \left(\frac{1}{C_{t}^{2}}\right), & C_{t} \neq 0 \\
0, & C_{t}=0,
\end{array}\right. \\
X_{0}=0, \quad \int_{0}^{t} \frac{\partial h}{\partial t}\left(s, C_{s}\right) \mathrm{d} s=0,
\end{gathered}
$$

and

$$
\int_{0}^{t} \frac{\partial h}{\partial x}\left(s, C_{s}\right) \mathrm{d} C_{s}=\int_{0}^{t} 2 C_{s} \sin \left(\frac{1}{C_{s}}\right)-\cos \left(\frac{1}{C_{s}}\right) \mathrm{d} C_{s}=\infty,
$$

since $2 x \sin \left(1 / x^{2}\right)-(2 / x) \cos \left(1 / x^{2}\right)$ is unbounded. That is,

$$
X_{t} \neq X_{0}+\int_{0}^{t} \frac{\partial h}{\partial t}\left(s, C_{s}\right) \mathrm{d} s+\int_{0}^{t} \frac{\partial h}{\partial x}\left(s, C_{s}\right) \mathrm{d} C_{s},
$$

which is equivalent to

$$
\mathrm{d} X_{t} \neq \frac{\partial h}{\partial t}\left(t, C_{t}\right) \mathrm{d} t+\frac{\partial h}{\partial x}\left(t, C_{t}\right) \mathrm{d} C_{t}
$$

When there exist multiple fuzzy processes in a dynamic system simultaneously, how do we compute the fuzzy differential? Theorem 4.2, Theorem 4.3 and Theorem 4.4 will answer this question. Here, we use the notation $\mathrm{d} \boldsymbol{Y}_{t}$ to denote $\left(\mathrm{d} Y_{1 t}, \mathrm{~d} Y_{2 t}, \cdots, \mathrm{d} Y_{p t}\right)^{T}$.

Theorem 4.2. Let $C_{t}$ be a standard Liu process, and let $\boldsymbol{h}(t, x)=\left(h_{1}(t, x)\right.$, $\left.h_{2}(t, x), \cdots, h_{p}(t, x)\right)^{T}$, where $h_{i}(t, x), i=1,2, \cdots, p$ are continuously differentiable functions. If fuzzy process $X_{t}$ is given by $\mathrm{d} X_{t}=u_{t} \mathrm{~d} t+v_{t} \mathrm{~d} C_{t}$, where 
$u_{t}, v_{t}$ are absolutely integrable fuzzy process and Liu integrable fuzzy process, respectively. Define $\boldsymbol{Y}_{t}=\boldsymbol{h}\left(t, X_{t}\right)$. Then

$$
\mathrm{d} \boldsymbol{Y}_{t}=\left(\begin{array}{c}
\mathrm{d} Y_{1 t} \\
\mathrm{~d} Y_{2 t} \\
\vdots \\
\mathrm{d} Y_{p t}
\end{array}\right)=\left(\begin{array}{c}
\frac{\partial h_{1}}{\partial t}\left(t, X_{t}\right) \mathrm{d} t+\frac{\partial h_{1}}{\partial x}\left(t, X_{t}\right) \mathrm{d} X_{t} \\
\frac{\partial h_{2}}{\partial t}\left(t, X_{t}\right) \mathrm{d} t+\frac{\partial h_{2}}{\partial x}\left(t, X_{t}\right) \mathrm{d} X_{t} \\
\vdots \\
\frac{\partial h_{p}}{\partial t}\left(t, X_{t}\right) \mathrm{d} t+\frac{\partial h_{p}}{\partial x}\left(t, X_{t}\right) \mathrm{d} X_{t}
\end{array}\right) .
$$

Proof. Clearly, $Y_{i t}=h_{i}\left(t, X_{t}\right)$ for each $i$ by the assumption of $\boldsymbol{Y}_{t}$. It follows from Liu formula that

$$
\mathrm{d} Y_{i t}=\frac{\partial h_{i}}{\partial t}\left(t, X_{t}\right) \mathrm{d} t+\frac{\partial h_{i}}{\partial x}\left(t, X_{t}\right) \mathrm{d} X_{t}
$$

The theorem is proved.

Next, $m$-dimensional Liu process is taken into consideration.

Theorem 4.3. Let $\left(C_{1 t}, C_{2 t}, \cdots, C_{m t}\right)^{T}$ be an m-dimensional standard Liu process, and let $h\left(t, x_{1}, x_{2}, \cdots, x_{n}\right)$ be a multivariate continuously differentiable function. If fuzzy process $\left(X_{1 t}, X_{2 t}, \cdots, X_{n t}\right)^{T}$ is given by

$$
\left\{\begin{array}{c}
\mathrm{d} X_{1 t}=u_{1 t} \mathrm{~d} t+v_{11 t} \mathrm{~d} C_{1 t}+\cdots+v_{1 m t} \mathrm{~d} C_{m t} \\
\vdots \\
\mathrm{d} X_{n t}=u_{n t} \mathrm{~d} t+v_{n 1 t} \mathrm{~d} C_{1 t}+\cdots+v_{n m t} \mathrm{~d} C_{m t}
\end{array}\right.
$$

where $u_{i}, v_{i j}$ are absolutely integrable fuzzy processes and Liu integrable fuzzy processes, respectively. Define $Y_{t}=h\left(t, X_{1 t}, X_{2 t}, \cdots, X_{n t}\right)$. Then

$$
\mathrm{d} Y_{t}=\frac{\partial h}{\partial t}\left(t, X_{1 t}, X_{2 t}, \cdots, X_{n t}\right) \mathrm{d} t+\sum_{i=1}^{n} \frac{\partial h}{\partial x_{i}}\left(t, X_{1 t}, X_{2 t}, \cdots, X_{n t}\right) \mathrm{d} X_{i t} .
$$

Proof. Denote $\boldsymbol{X}_{t}=\left(X_{1 t}, X_{2 t}, \cdots, X_{n t}\right)^{T}$. Indeed, each $X_{i s}$ is a continuous fuzzy process by Theorem 3.2. Thus each $X_{i s}$ is bounded on $[0, t]$, i.e. there exist $M_{i}>0$, such that $\left|X_{i s}\right| \leq M_{i}$ for all $s \in[0, t]$ and $i=1,2, \cdots, n$. As continuous functions, $\partial h / \partial t$ and $\partial h / \partial x_{i}$ are uniformly continuous and bounded on $[0, t] \times\left[-M_{1}, M_{1}\right] \times \cdots \times\left[-M_{n}, M_{n}\right]$ for all $i$. It follows from Taylor's theorem that

$$
\begin{aligned}
h\left(t, \boldsymbol{X}_{t}^{T}\right) & =h\left(0, \boldsymbol{X}_{0}^{T}\right)+\sum_{j} \triangle h\left(t_{j}, \boldsymbol{X}_{t_{j}}^{T}\right) \\
& =h\left(0, \boldsymbol{X}_{0}^{T}\right)+\sum_{j} \frac{\partial h}{\partial t} \triangle t_{j}+\sum_{i=1}^{n} \sum_{j} \frac{\partial h}{\partial x_{i}} \triangle X_{i j}+\sum_{j} R_{j},
\end{aligned}
$$


where $\partial h / \partial t, \partial h / \partial x_{i}$ etc. are evaluated at the points $\left(t_{j}, X_{1 t_{j}}, X_{2 t_{j}}, \cdots, X_{n t_{j}}\right)$,

$$
\begin{gathered}
\triangle t_{j}=t_{j+1}-t_{j}, \quad R_{j}=o\left(\sqrt{\left|\triangle t_{j}\right|^{2}+\sum_{i=1}^{n}\left|\triangle X_{i j}\right|^{2}}\right), \\
\triangle X_{i j}=X_{i t_{j+1}}-X_{i t_{j}}, \Delta h\left(t_{j}, \boldsymbol{X}_{t_{j}}^{T}\right)=h\left(t_{j+1}, \boldsymbol{X}_{t_{j+1}}^{T}\right)-h\left(t_{j}, \boldsymbol{X}_{t_{j}}^{T}\right)
\end{gathered}
$$

for all $i$ and $j$. Denote $\triangle=\sup _{j} \Delta t_{j}$. If $\triangle \rightarrow 0$, it follows from the definition of Riemann integral that

$$
\sum_{j} \frac{\partial h}{\partial t} \triangle t_{j}=\sum_{j} \frac{\partial h}{\partial t}\left(t_{j}, \boldsymbol{X}_{t_{j}}^{T}\right) \triangle t_{j} \rightarrow \int_{0}^{t} \frac{\partial h}{\partial t}\left(s, \boldsymbol{X}_{s}^{T}\right) \mathrm{d} s \quad \text { a.s. }
$$

Since $\partial h / \partial x_{i}\left(t, \boldsymbol{X}_{t}^{T}\right)$ are continuous functions, it follows from Theorem 3.1 that

$$
\sum_{j} \frac{\partial h}{\partial x_{i}} \triangle X_{i j}=\sum_{j} \frac{\partial h}{\partial x_{i}}\left(t_{j}, \boldsymbol{X}_{t_{j}}^{T}\right) \triangle X_{i j} \rightarrow \int_{0}^{t} \frac{\partial h}{\partial x_{i}}\left(s, \boldsymbol{X}_{s}^{T}\right) \mathrm{d} X_{i s} \quad \text { a.s. }
$$

When $\triangle t_{j} \rightarrow 0$, we have $\sum_{j} R_{j} \rightarrow 0$.

In fact, since

$$
R_{j}=o\left(\sqrt{\left|\triangle t_{j}\right|^{2}+\sum_{i=1}^{n}\left|\triangle X_{i j}\right|^{2}}\right),
$$

there exist $\alpha_{j}=o\left(\triangle t_{j}\right)$ and $\beta_{i j}=o\left(\triangle X_{i j}\right)$ such that $R_{j}=\alpha_{j} \triangle t_{j}+\sum_{i=1}^{n} \beta_{i j} \triangle X_{i j}$.

Then

$$
\triangle X_{i j}=X_{i t_{j+1}}-X_{i t_{j}}=u_{i t_{j}} \triangle t_{j}+\sum_{k=1}^{m} v_{i k t_{j}} \triangle C_{k t_{j}} \rightarrow 0,
$$

when $\triangle \rightarrow 0$.

Thus, we have

$$
\begin{aligned}
\left|\sum_{j} R_{j}\right| & =\left|\sum_{j}\left(\alpha_{j} \triangle t_{j}+\sum_{i=1}^{n} \beta_{i j} \triangle X_{i j}\right)\right| \\
& \leq \sum_{j}\left|\alpha_{j}\right| \triangle t_{j}+\sum_{j} \sum_{i=1}^{n}\left|\beta_{i j}\right|\left|\triangle X_{i j}\right| \\
& \leq \sum_{j}\left|\alpha_{j}\right| \triangle t_{j}+\sum_{i=1}^{n} \sum_{j}\left|\beta_{i j}\right|\left|u_{i t_{j}}\right| \triangle t_{j}+\sum_{i=1}^{n} \sum_{k=1}^{m} \sum_{j}\left|v_{i k t_{j}}\right|\left|\beta_{i j}\right|\left|\triangle C_{k t_{j}}\right| \\
& \rightarrow 0,
\end{aligned}
$$


when $\triangle \rightarrow 0$. Hence

$$
h\left(t, \boldsymbol{X}_{t}^{T}\right)=h\left(0, \boldsymbol{X}_{0}^{T}\right)+\int_{0}^{t} \frac{\partial h}{\partial t}\left(s, \boldsymbol{X}_{s}^{T}\right) \mathrm{d} s+\sum_{i=1}^{n} \int_{0}^{t} \frac{\partial h}{\partial x_{i}}\left(s, \boldsymbol{X}_{s}^{T}\right) \mathrm{d} X_{i s},
$$

which is equivalent to the expression

$$
\mathrm{d} X_{t}=\frac{\partial h}{\partial t}\left(t, X_{1 t}, X_{2 t}, \cdots, X_{n t}\right) \mathrm{d} t+\sum_{i=1}^{n} \frac{\partial h}{\partial x_{i}}\left(t, X_{1 t}, X_{2 t}, \cdots, X_{n t}\right) \mathrm{d} X_{i t}
$$

Example 4.2: Let $\boldsymbol{C}_{t}=\left(C_{1 t}, C_{2 t}, \cdots, C_{m t}\right)^{T}$ be an $m$-dimensional standard Liu process. Fuzzy processes $X_{1 t}$ and $X_{2 t}$ are given by

$$
\left\{\begin{array}{l}
\mathrm{d} X_{1 t}=u_{1 t} \mathrm{~d} t+v_{11 t} \mathrm{~d} C_{1 t}+\cdots+v_{1 m t} \mathrm{~d} C_{m t} \\
\mathrm{~d} X_{2 t}=u_{2 t} \mathrm{~d} t+v_{21 t} \mathrm{~d} C_{1 t}+\cdots+v_{2 m t} \mathrm{~d} C_{m t}
\end{array},\right.
$$

where $u_{i t}, v_{i j t}$ are absolutely integrable fuzzy processes and Liu integrable fuzzy processes, respectively. Then we have

$$
\mathrm{d}\left(X_{1 t} X_{2 t}\right)=X_{1 t} \mathrm{~d} X_{2 t}+X_{2 t} \mathrm{~d} X_{1 t} .
$$

More generally, in a fuzzy dynamic system, when there exist multiple fuzzy processes and the Liu process concerned is multi-dimensional, in this case, we have multi-dimensional Liu formula.

Theorem 4.4. (Multi-dimensional Liu Formula) Let $\boldsymbol{C}_{t}=\left(C_{1 t}, C_{2 t}, \cdots, C_{m t}\right)^{T}$ be an $m$-dimensional standard Liu process, and let $\boldsymbol{h}\left(t, x_{1}, x_{2}, \cdots, x_{n}\right)=\left(h_{1}(t\right.$, $\left.\left.x_{1}, x_{2}, \cdots, x_{n}\right), h_{2}\left(t, x_{1}, x_{2}, \cdots, x_{n}\right), \cdots, h_{p}\left(t, x_{1}, x_{2}, \cdots, x_{n}\right)\right)^{T}$ where $h_{i}\left(t, x_{1}\right.$, $\left.x_{2}, \cdots, x_{n}\right), i=1,2, \cdots, p$ are multivariate continuously differentiable functions. If fuzzy process $\left(X_{1 t}, X_{2 t}, \cdots, X_{n t}\right)^{T}$ is given by

$$
\left\{\begin{array}{c}
\mathrm{d} X_{1 t}=u_{1 t} \mathrm{~d} t+v_{11 t} \mathrm{~d} C_{1 t}+\cdots+v_{1 m t} \mathrm{~d} C_{m t} \\
\vdots \\
\vdots \\
\mathrm{d} X_{n t}=u_{n t} \mathrm{~d} t+v_{n 1 t} \mathrm{~d} C_{1 t}+\cdots+v_{n m t} \mathrm{~d} C_{m t}
\end{array}\right.
$$


where $u_{i t}, v_{i j t}$ are absolutely integrable fuzzy processes and Liu integrable fuzzy processes, respectively. Define $\boldsymbol{Y}_{t}=\boldsymbol{h}\left(t, X_{1 t}, X_{2 t}, \cdots, X_{n t}\right)$. Then

$$
\mathrm{d} \boldsymbol{Y}_{t}=\left(\begin{array}{c}
\frac{\partial h_{1}}{\partial t}\left(t, X_{1 t}, X_{2 t}, \cdots, X_{n t}\right) \mathrm{d} t+\sum_{i=1}^{n} \frac{\partial h_{1}}{\partial x_{i}}\left(t, X_{1 t}, X_{2 t}, \cdots, X_{n t}\right) \mathrm{d} X_{i t} \\
\frac{\partial h_{2}}{\partial t}\left(t, X_{1 t}, X_{2 t}, \cdots, X_{n t}\right) \mathrm{d} t+\sum_{i=1}^{n} \frac{\partial h_{2}}{\partial x_{i}}\left(t, X_{1 t}, X_{2 t}, \cdots, X_{n t}\right) \mathrm{d} X_{i t} \\
\vdots \\
\frac{\partial h_{p}}{\partial t}\left(t, X_{1 t}, X_{2 t}, \cdots, X_{n t}\right) \mathrm{d} t+\sum_{i=1}^{n} \frac{\partial h_{p}}{\partial x_{i}}\left(t, X_{1 t}, X_{2 t}, \cdots, X_{n t}\right) \mathrm{d} X_{i t}
\end{array}\right) .
$$

Proof. Since $\boldsymbol{Y}_{t}=\boldsymbol{h}\left(t, X_{1 t}, X_{2 t}, \cdots, X_{n t}\right), \quad Y_{j t}=h_{j}\left(t, X_{1 t}, X_{2 t}, \cdots, X_{n t}\right)$ for each $j$. It follows from Theorem 4.2 and Theorem 4.3 that

$$
\mathrm{d} Y_{j t}=\frac{\partial h_{j}}{\partial t}\left(t, X_{1 t}, X_{2 t}, \cdots, X_{n t}\right) \mathrm{d} t+\sum_{i=1}^{n} \frac{\partial h_{j}}{\partial x_{i}}\left(t, X_{1 t}, X_{2 t}, \cdots, X_{n t}\right) \mathrm{d} X_{i t} .
$$

The theorem is proved.

Example 4.3: Let $\boldsymbol{C}_{t}=\left(C_{1 t}, C_{2 t}, C_{3 t}\right)^{T}$ be a 3 -dimensional standard Liu process, and let $\boldsymbol{X}_{t}=\left(t+C_{1 t}+C_{2 t}+C_{3 t}, C_{2 t}^{2}-C_{1 t} C_{3 t}\right)^{T}$. It follows from multi-dimensional Liu formula that

$$
\mathrm{d} \boldsymbol{X}_{t}=\left(\begin{array}{l}
\mathrm{d} X_{1 t} \\
\mathrm{~d} X_{2 t}
\end{array}\right)=\left(\begin{array}{c}
\mathrm{d} t+\mathrm{d} C_{1 t}+\mathrm{d} C_{2 t}+\mathrm{d} C_{3 t} \\
-C_{3 t} \mathrm{~d} C_{1 t}+2 C_{2 t} \mathrm{~d} C_{2 t}-C_{1 t} \mathrm{~d} C_{3 t}
\end{array}\right) .
$$

\section{Conclusions}

In this paper, multi-dimensional fuzzy process was proposed, especially the multi-dimensional Liu process. Meanwhile, Liu integral and Liu formula were extended to multi-dimensional case.

\section{References}

[1] J. J. Buckley, and T. Feuring, Fuzzy differential equations, Fuzzy Sets and Systems 110(2000), 43-54.

[2] W. Dai, Reflection principle of Liu process, http://orsc.edu.cn/process /071110.pdf.

[3] Z. Ding, M. Ma and A. Kandel, Existence of the solutions of fuzzy differential equations with parameters, Information Sciences 99(1999), no. 3-4, 1205-1217.

[4] J Gao, Credibiltistic option pricing: A new model, http://orsc.edu.cn/process /071124.pdf.

[5] X Gao, Option pricing formula for Gao's stock model, http://orsc.edu.cn/ process/080326.pdf.

[6] X Gao X, and X Chen, Option pricing formula for generalized stock models, http://orsc.edu.cn /process/080317.pdf.

[7] O. Kaleva, Fuzzy differential equations, Fuzzy Sets and Systems 24(1987), no. 3, 301317. 
[8] O. Kaleva, The Cauchy problem for fuzzy differential equations, Fuzzy Sets and Systems 35(1987), no. 3, 389-396.

[9] O. Kaleva, A note on fuzzy differential equations, Nonlinear Analysis 64(2006), no. 5, 895-900.

[10] X. Li, and B. Liu, A sufficent and necessary condition for credibility measures, International Journal of Uncertainty, Fuzziness \& Knowledge-Based Systems 14(2006), no. 5, 527-535.

[11] B. Liu, Uncertainty Theory, Springer-Verlag, Berlin, 2004.

[12] B. Liu, Uncertainty Theory, 2nd ed., Springer-Verlag, Berlin, 2007.

[13] B. Liu, Fuzzy process, hybrid process and uncertain process, Journal of Uncertain Systems 2(2008), no. 1, 3-16.

[14] B. Liu B, and Y. K. Liu, Expected value of fuzzy variable and fuzzy expected value models, IEEE Transactions on Fuzzy Systems 10(2002), no. 4, 445-450.

[15] V. Lakshmikantham, and R. N. Mohapatra, Theory of fuzzy differentials equations and inclusions, Taylor \& Francis, London, 2003.

[16] Pearson DW, A property of linear fuzzy differential equations, Applied Mathematics Letters 14(1997), no. 3, 99-103.

[17] Peng J, A general stock model for fuzzy markets, Journal of uncertain Systems 2(2008), no. 4, 248-254.

[18] Qin Z, On analytic functions of complx Liu process, http://orsc.edu.cn/process /071026.pdf.

[19] Qin Z, A new fuzzy control system with application to production planning problem, http:// orsc.edu.cn/process/080412.pdf.

[20] Qin Z, and Li X, Option pricing formula for fuzzy financial market, Journal of uncertain Systems 2(2008), no. 1, 17-21.

[21] Qin Z, and Li X, Expected payoff of trading strategies iInvolving European options for fuzzy financial market, http://orsc.edu.cn/process/080407.pdf.

[22] L. A. Zadeh, Fuzzy sets, Information and Control 8(1965), 338-353.

[23] Y. Zhu, Fuzzy optimal control with application to portfolio selection, http://orsc.edu.cn /process /080117.pdf.

Cuilian You

College of Mathematics and Computer Science, Hebei University, Baoding 071002, CHINA

E-mail address: yycclian@163.com

Huae Huo

College of Mathematics and Computer Science, Hebei University, Baoding 071002, CHINA

E-mail address: huohuaehhe@163.com

WEIQING WANG

College of Mathematics and Computer Science, Hebei University, Baoding 071002, CHINA

E-mail address: wangweiqing.007@163.com 